\title{
Isolation of 2019-nCoV from a Stool Specimen of a Laboratory- Confirmed Case of the Coronavirus Disease 2019 (COVID-19)
}

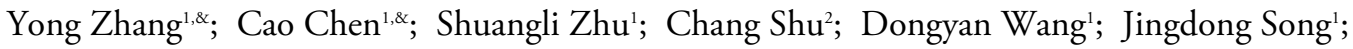 \\ Yang Song'; Wei Zhen'; Zijian Feng'; Guizhen $\mathrm{Wu}^{1}$; Jun $\mathrm{Xu}^{2, * *}$; Wenbo $\mathrm{Xu}^{1, *}$
}

The novel coronavirus (2019-nCoV) is spreading very fast in Hubei Province of China. As of February 14, 2020, 51,986 confirmed cases (including laboratory-confirmed cases and clinically-confirmed cases) were reported in Hubei Province, and 1,318 of them died. Respiratory droplets and contact transmission are considered to be the most important routes of transmission of 2019-nCoV, but do not fully account for the occurrence of all coronavirus disease 2019 (COVID-19) cases, previously known as novel coronavirus pneumonia $(\mathrm{NCP})$, and the reasons for the rapid spread of this virus $(1)$.

In Biosafety Level 3 (BSL-3) Laboratory of the
National Institute for Viral Disease Control and Prevention, Vero cells were used for viral isolation from stool samples of COVID-19 patients sent by Heilongjiang CDC. A 2019-nCoV strain was isolated from a stool specimen of a laboratory-confirmed COVID-19 severe pneumonia case, who experienced onset on January 16, 2020 and was sampled on February 1, 2020. The interval between sampling and onset was 15 days. The full-length genome sequence indicated that the virus had high-nucleotide similarity $(99.98 \%)$ to that of the first isolated novel coronavirus isolated from Wuhan, China (Figure 1). In the Vero cells, viral particles with typical morphology of a

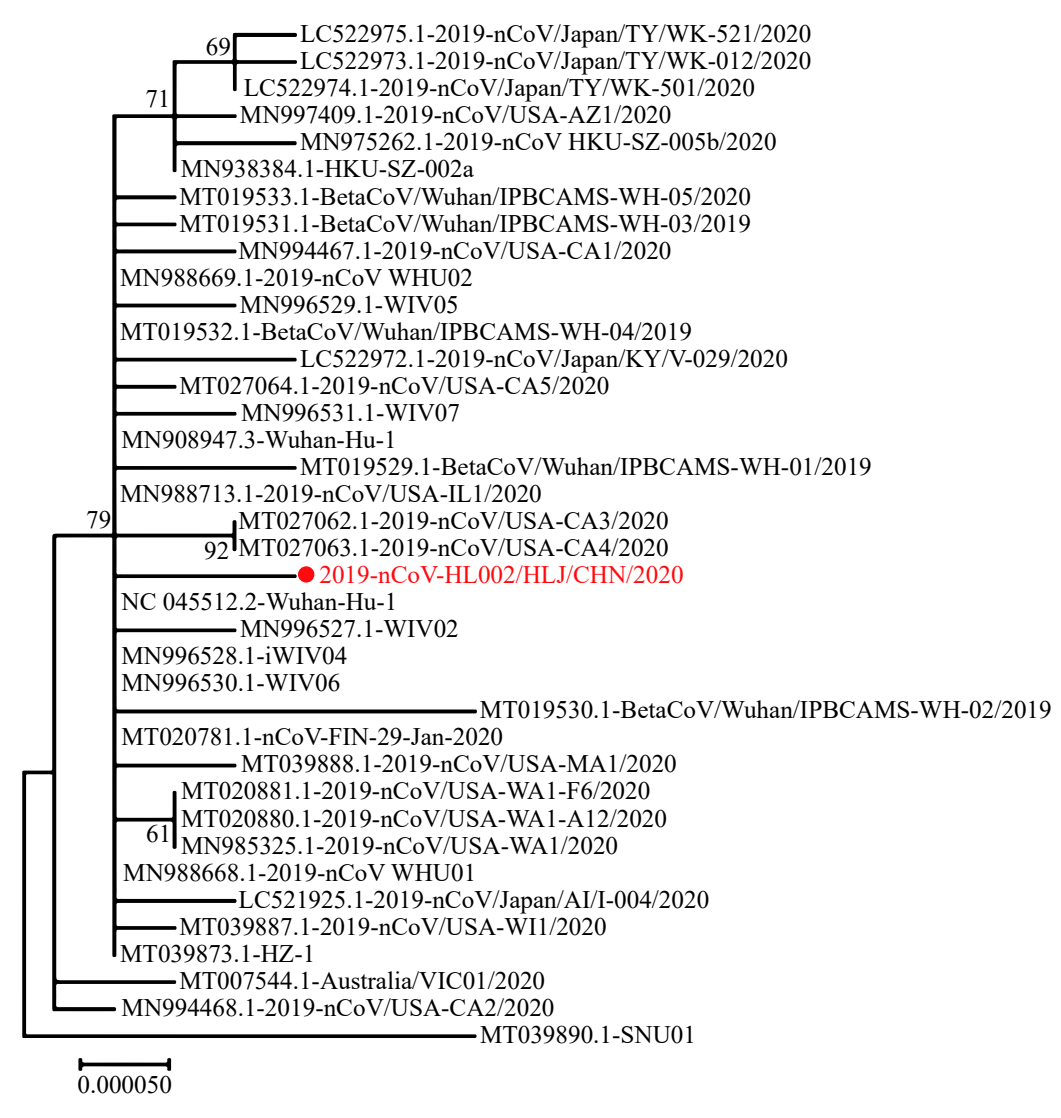

FIGURE 1. Phylogenetic tree based on the full-length genome sequences of 2019-nCoV. The sequence represented by the red circle is the Heilongjiang sequence in this study. 
coronavirus could be observed under the electron microscope (Figure 2).

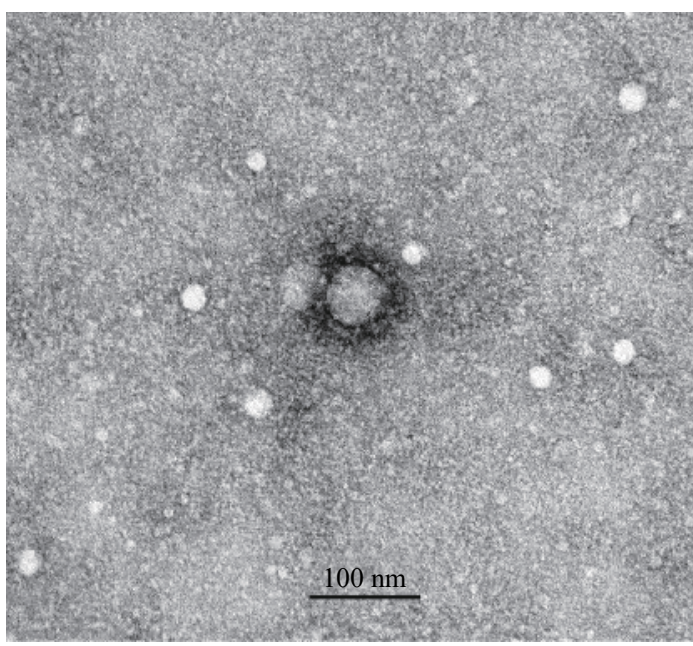

FIGURE 2. A virus particle with typical morphology of coronavirus was observed using electron microscopy after inoculating stool suspension into Vero cells.

These results confirm that COVID-19 patients have live virus in stool specimens, which is a new finding in the transmission routes of 2019-nCoV. In addition to close contact and contact with respiratory secretions of patients, the virus can also be transmitted through the potential fecal-oral route. This means that stool samples may contaminate hands, food, water, etc., and may cause infection by invading the oral cavity, respiratory mucosa, conjunctiva, etc. This virus has many routes of transmission, which can partially explain its strong transmission and fast transmission speed. This study also verified that the nucleic acids of 2019-nCoV can be detected from stool samples (2).

This finding has important public health significance. Suggestions to strengthen the control of fecal oral transmission of 2019-nCoV include strengthening health publicity and education; maintaining environmental health and personal hygiene; drinking boiled water, avoiding raw food consumption, and implementing separate meal systems in epidemic areas; frequently washing hands and disinfecting of surfaces of objects in households, toilets, public places, and transportation vehicles; and disinfecting the excreta and environment of patients in medical facilities to prevent water and food contamination from patients' stool samples.

\section{Acknowledgements}

This study was supported by the National Key Technology R\&D Programs of China (Project Nos. 2018ZX10713002 and 2017ZX10104001).

\# Corresponding authors: Jun Xu, 1359865696@ qq.com; Wenbo Xu, xuwb@ivdc.chinacdc.cn.

\footnotetext{
${ }^{1}$ National Health Commission Key Laboratory for Medical Virology, National Institute for Viral Disease Control and Prevention, Chinese Center for Disease Control and Prevention, Beijing, China; Heilongjiang Center for Disease Control and Prevention, Haerbin, China; ${ }^{3}$ Chinese Center for Disease Control and Prevention, Beijing, China.

\& Joint first authors.

Submitted: February 14, 2020; Accepted: February 14, 2020
}

\section{References}

1. Riou J, Althaus CL. Pattern of early human-to-human transmission of Wuhan 2019 novel coronavirus (2019-nCoV), December 2019 to January 2020. Euro Surveill 2020;25(4). http://dx.doi.org/10.2807/ 1560-7917.ES.2020.25.4.2000058.

2. Holshue ML, DeBolt C, Lindquist S, Lofy KH, Wiesman J, Bruce H, et al. First case of 2019 novel coronavirus in the United States. N Engl J Med. http://dx.doi.org/10.1056/NEJMoa2001191. [2020-01-30]. 